\title{
Robust Control for Discrete-Time Networked Control Systems
}

\author{
Dongxiao Wu, Jun $\mathrm{Wu}$ and Sheng Chen
}

\begin{abstract}
This paper considers analysis and synthesis of discrete-time networked control systems (NCSs), where the plant has additive uncertainty and the controller is updated with the sensor information at stochastic time intervals. It is shown that the problem is linked to robust control of linear discrete-time stochastic systems and a new small gain theorem is established. Based on this result, sufficient conditions are given for ensuring mean square stability of the NCS, and the genetic algorithm is utilised to design the controller of the NCS based on a linear matrix inequality technique.
\end{abstract}

\section{INTRODUCTION}

Networked control systems (NCSs) have received much attention recently [1]- [5]. A NCS is the system in which a control loop is closed via a shared communication network. The use of a shared network in the feedback path has advantages of low installation cost, reducing system wiring, simple system diagnosis and easy maintenance. However, some inherent shortcomings, such as bandwidth constraints, packet delays and packet dropouts, will degrade performance of NCSs or even cause instability. Stability analysis of NCSs is investigated in [6]- [9], and stabilising controllers are designed in [10], [11]. Typically stochastic approaches are adopted to deal with network packet dropout and to establish the stability of the NCS in the sense of mean square statistics [12]- [15]. The works of [15]- [18] adopt robust control theory for the analysis and design of NCSs.

Most of the works in the NCS research utilise fixed controllers. Some exceptions are [7]- [9], which adopt more flexible controllers for NCSs where a network is located between the sensor and the controller. For NCSs, time periods frequently appear during which the controller cannot access sensor data due to network induced random delay and packet dropout. During these periods without sensor data, the underlying idea of [7]- [9] is that a nominal plant model is employed at the controller to estimate the plant behaviour, and the estimated result is provided to the controller to replace the real plant behaviour information so that the computation of control signal can be executed in time. During time periods when the controller can access sensor data, the networked controllers perform the same feedback control as standard closedloop control systems without network. This kind of control scheme is referred to as model-based networked control by [7],

D.-X. Wu and J. Wu are with State Key Laboratory of Industrial Control Technology, Institute of Cyber-Systems and Control, Zhejiang University, Hangzhou 310027, China.

S. Chen is with School of Electronics and Computer Science, University of Southampton, Southampton SO17 1BJ, UK.

This work is supported by National Natural Science Foundation of China (Grants No.60774001, No.60736021 and No.60721062), 973 Program of China (Grant No.2009CB320603), 863 Program of China (Grant No.2008AA042602), 111 Project of China (Grant No.B07031); UK Royal Society and Royal Academy of Engineering.
[8], where model-based networked state-feedback and modelbased networked observed-state-feedback control methods are presented. The smart control of NCSs addressed in [9] is another model-based networked observed-state-feedback control method. In the networked control schemes of [7], [8], the observer is included at the actuator side of the plant to be controlled, while for the networked control scheme of [9] the observer is located at the controller side.

All the plant parameters are assumed to be known in the works [7], [8] while the plant is assumed to be uncertainty free in [9]. But these assumptions on the plant are not met in practice. It is important to remove these strict assumptions on the plant and to study model-based networked control with robustness considerations. Also the study by [9] on smart control does not consider the networks of random transmission with known probability, which belong to a most important class of networks in NCSs. The novel contribution of this paper is that we study smart control for NCSs where discretetime plants have additive perturbations and networks induce random delay and packet dropout. To our best knowledge, our key result, Theorem 1, which establishes a new small gain theorem for linear discrete-time stochastic systems, was not seen previously in the literature.

\section{NOTATIONS AND PREliminary RESUlts}

Let $\mathbb{R}$ stand for real numbers and $\mathbb{N}$ for nonnegative integers. $\mathbf{I}_{n}$ denotes the $n \times n$ identity matrix, while $\mathbf{I}$ and $\mathbf{0}$ are the identity and zero matrices of appropriate dimensions, respectively. For $\mathbf{M} \in \mathbb{R}^{m \times n}$ and $p \geq m$, denote

$$
\mathcal{H}_{p}(\mathbf{M})= \begin{cases}\mathbf{M} \in \mathbb{R}^{p \times n}, & p=m, \\
{\left[\begin{array}{c}
\mathbf{M} \\
\mathbf{0}
\end{array}\right] \in \mathbb{R}^{p \times n},} & p>m .\end{cases}
$$

Similarly, for $\mathbf{M} \in \mathbb{R}^{m \times n}$ and $q \geq n$, denote

$$
\mathcal{W}_{q}(\mathbf{M})= \begin{cases}\mathbf{M} \in \mathbb{R}^{m \times q}, & q=n, \\
{\left[\begin{array}{ll}
\mathbf{M} & \mathbf{0}
\end{array}\right] \in \mathbb{R}^{m \times q},} & q>n .\end{cases}
$$

For $\mathbf{S} \in \mathbb{R}^{m \times m}, \mathbf{S}>0(\geq 0)$ indicates that $\mathbf{S}$ is a positive definite (semidefinite) matrix. For symmetric $\mathbf{S}_{1} \in \mathbb{R}^{m \times m}$ and $\mathbf{S}_{2} \in \mathbb{R}^{m \times m}, \mathbf{S}_{1}>\mathbf{S}_{2}$ means that $\mathbf{S}_{1}-\mathbf{S}_{2}>0$.

For a discrete-time signal $\mathbf{r}=\{\mathbf{r}(t)\}_{t \in \mathbb{N}}$ with $\mathbf{r}(t) \in \mathbb{R}^{r}$, define

$$
\|\mathbf{r}\|_{2} \triangleq \sqrt{\sum_{t=0}^{\infty} \mathbf{r}^{\mathrm{T}}(t) \mathbf{r}(t)}
$$

Let $\ell_{2}^{r}$ be the set of $\mathbf{r s}$ with $\|\mathbf{r}\|_{2}<\infty$. A finite-dimensional linear time-invariant discrete-time system $\hat{G}$ is written as

$$
\left\{\begin{array}{l}
\mathbf{x}_{g}(t+1)=\mathbf{A}_{g} \mathbf{x}_{g}(t)+\mathbf{B}_{g} \mathbf{u}_{g}(t), \quad t \in \mathbb{N} \\
\mathbf{y}_{g}(t)=\mathbf{C}_{g} \mathbf{x}_{g}(t)+\mathbf{D}_{g} \mathbf{u}_{g}(t),
\end{array}\right.
$$


where $\mathbf{x}_{g}(t) \in \mathbb{R}^{b}, \mathbf{u}_{g}(t) \in \mathbb{R}^{r}$ and $\mathbf{y}_{g}(t) \in \mathbb{R}^{d}$ are state, input and output, respectively; $\mathbf{A}_{g}, \mathbf{B}_{g}, \mathbf{C}_{g}$ and $\mathbf{D}_{g}$ are constant real matrices of appropriate dimensions. $\hat{G}$ with $\mathbf{u}_{g}(t) \equiv \mathbf{0}$ is stable if $\forall \mathbf{x}_{g}(0) \in \mathbb{R}^{b}, \lim _{t \rightarrow \infty} \mathbf{x}_{g}^{\mathrm{T}}(t) \mathbf{x}_{g}(t)=0$. The $\mathrm{H}_{\infty}$-norm of $\hat{G}$ is defined as

$$
\|\hat{G}\|_{\infty} \triangleq \sup _{\substack{\mathbf{u}_{g} \in \ell_{2}^{r} \\\left\|\mathbf{u}_{g}\right\|_{2} \neq 0 \\ \mathbf{x}_{g}(0)=\mathbf{0}}} \frac{\left\|\mathbf{y}_{g}\right\|_{2}}{\left\|\mathbf{u}_{g}\right\|_{2}} .
$$

For $0<\rho \in \mathbb{R}$, let $\mathbb{D}_{\rho}^{d \times r}$ be the set of $\hat{G}$ s, which are described by (4) with $\|\hat{G}\|_{\infty}<1 / \rho$, and are stable for $\mathbf{u}_{g}(t) \equiv \mathbf{0}$.

Lemma 1: (See [19]) The system $\hat{G} \in \mathbb{D}_{\rho}^{d \times r}$ if and only if there exists a $0<\mathbf{P} \in \mathbb{R}^{b \times b}$ such that

$$
\left[\begin{array}{cc}
\mathbf{P} & \mathbf{0} \\
\mathbf{0} & \frac{1}{\rho^{2}} \mathbf{I}_{r}
\end{array}\right]-\left[\begin{array}{cc}
\mathbf{A}_{g} & \mathbf{B}_{g} \\
\mathbf{C}_{g} & \mathbf{D}_{g}
\end{array}\right]^{\mathrm{T}}\left[\begin{array}{cc}
\mathbf{P} & \mathbf{0} \\
\mathbf{0} & \mathbf{I}_{d}
\end{array}\right]\left[\begin{array}{cc}
\mathbf{A}_{g} & \mathbf{B}_{g} \\
\mathbf{C}_{g} & \mathbf{D}_{g}
\end{array}\right]
$$$$
>0 \text {. }
$$

For a discrete-time stochastic signal $\overline{\mathbf{r}}=\{\overline{\mathbf{r}}(t)\}_{t \in \mathbb{N}}$ with $\overline{\mathbf{r}}(t)$ a $\mathbb{R}^{r}$-valued random variable, define

$$
\|\overline{\mathbf{r}}\|_{2 s} \triangleq \sqrt{\sum_{t=0}^{\infty} E\left(\overline{\mathbf{r}}^{\mathrm{T}}(t) \overline{\mathbf{r}}(t)\right)}
$$

where $E(\cdot)$ denotes the expectation. Let $\ell_{2 s}^{r}$ be the set of $\overline{\mathbf{r}}$ s with $\|\overline{\mathbf{r}}\|_{2 s}<\infty$. For positive integer $M$, denote $\mathcal{M}=$ $\{1, \cdots, M\}$. Consider the stochastic system $\hat{F}$ :

$$
\left\{\begin{array}{l}
\mathbf{x}_{f}(t+1)=\mathbf{A}_{f}\left(\theta_{t}\right) \mathbf{x}_{f}(t)+\mathbf{B}_{f}\left(\theta_{t}\right) \mathbf{u}_{f}(t), \quad t \in \mathbb{N} \\
\mathbf{y}_{f}(t)=\mathbf{C}_{f}\left(\theta_{t}\right) \mathbf{x}_{f}(t)+\mathbf{D}_{f}\left(\theta_{t}\right) \mathbf{u}_{f}(t),
\end{array}\right.
$$

where $\theta_{t}$ s are i.i.d. $\mathcal{M}$-valued random variables; $\mathbf{A}_{f}, \mathbf{B}_{f}, \mathbf{C}_{f}$ and $\mathbf{D}_{f}$ are mappings from $\mathcal{M}$ to $\mathbb{R}^{b \times b}, \mathbb{R}^{b \times r}, \mathbb{R}^{d \times b}$ and $\mathbb{R}^{d \times r}$, respectively. The probability mass function of $\theta_{t}$ is given by $q_{j}=\operatorname{Pr}\left(\theta_{t}=j\right)$ with $j \in \mathcal{M}$. Clearly, $\theta_{t}$ can be regarded as a special Markov chain.

Lemma 2: (See [12], [15]) For the system $\hat{F}$ in (8), the following are equivalent:

1) $\hat{F}$ with $\mathbf{u}_{f}(t) \equiv \mathbf{0}$ is stochastically stable;

2) $\mathbf{A}_{f}\left(\theta_{t}\right)$ is stochastically stable;

3) $\forall \mathbf{x}_{f}(0) \in \mathbb{R}^{b}, \lim _{t \rightarrow \infty} E\left(\mathbf{x}_{f}^{\mathrm{T}}(t) \mathbf{x}_{f}(t)\right)=0$ with $\mathbf{u}_{f}(t) \equiv \mathbf{0}$

4) $\forall \mathbf{x}_{f}(0) \in \mathbb{R}^{b},\left\|\mathbf{x}_{f}\right\|_{2 s}^{2}<\infty$ with $\mathbf{u}_{f}(t) \equiv \mathbf{0}$;

5) there exists a $0<\mathbf{P} \in \mathbb{R}^{b \times b}$ such that $\mathbf{P}$ $\sum_{j=1}^{M} q_{j} \mathbf{A}_{f}^{\mathrm{T}}(j) \mathbf{P} \mathbf{A}_{f}(j)>0$.

$\left(\mathbf{C}_{f}\left(\theta_{t}\right), \mathbf{A}_{f}\left(\theta_{t}\right)\right)$ is said to be stochastically detectable if there exists a $\mathbf{H}_{f}\left(\theta_{t}\right)$ mapping $\mathcal{M}$ to $\mathbb{R}^{r \times b}$ such that $\mathbf{A}_{f}\left(\theta_{t}\right)-$ $\mathbf{H}_{f}\left(\theta_{t}\right) \mathbf{C}_{f}\left(\theta_{t}\right)$ is stochastically stable.

Lemma 3: (See [20]) Suppose that $\left(\mathbf{C}_{f}\left(\theta_{t}\right), \mathbf{A}_{f}\left(\theta_{t}\right)\right)$ is stochastically detectable. Then the following are equivalent:

1) $\mathbf{A}_{f}\left(\theta_{t}\right)$ is stochastically stable;

2) the discrete-time backward difference equations

$$
\mathbf{X}_{t}(j)=\mathbf{A}_{f}^{\mathrm{T}}(j) \sum_{l=1}^{M} q_{l} \mathbf{X}_{t+1}(l) \mathbf{A}_{f}(j)+\mathbf{C}_{f}^{\mathrm{T}}(j) \mathbf{C}_{f}(j),
$$

where $t \in \mathbb{N}$ and $j \in \mathcal{M}$, have a bounded solution $\left\{\left[\overline{\mathbf{X}}_{t}^{\mathrm{T}}(1) \cdots \overline{\mathbf{X}}_{t}^{\mathrm{T}}(M)\right]^{\mathrm{T}}\right\}_{t \in \mathbb{N}}$ such that $\forall t \in \mathbb{N}$ and $\forall j \in \mathcal{M}$, $0 \leq \overline{\mathbf{X}}_{t}(j) \in \mathbb{R}^{b \times b}$.
Replacing the backward difference equations with algebraic equations leads to Corollary 1.

Corollary 1: Suppose that $\left(\mathbf{C}_{f}\left(\theta_{t}\right), \mathbf{A}_{f}\left(\theta_{t}\right)\right)$ is stochastically detectable, and there exist $0 \leq \mathbf{X}(j) \in \mathbb{R}^{b \times b}, j \in \mathcal{M}$, satisfying

$$
\mathbf{X}(j)=\mathbf{A}_{f}^{\mathrm{T}}(j) \sum_{l=1}^{M} q_{l} \mathbf{X}(l) \mathbf{A}_{f}(j)+\mathbf{C}_{f}^{\mathrm{T}}(j) \mathbf{C}_{f}(j),
$$

with $j \in \mathcal{M}$. Then $\mathbf{A}_{f}\left(\theta_{t}\right)$ is stochastically stable.

The $\mathrm{H}_{\infty}$-norm of stochastic system $\hat{F}$ is defined as

$$
\|\hat{F}\|_{\infty s} \triangleq \sup _{\substack{\mathbf{u}_{f} \in \ell_{2 s}^{r} \\\left\|_{\mathbf{u}_{f}}\right\|_{2 s} \neq 0 \\ \mathbf{x}_{f}(0)=\mathbf{0} \\ \theta_{0} \in \mathcal{M}}} \frac{\left\|\mathbf{y}_{f}\right\|_{2 s}}{\left\|\mathbf{u}_{f}\right\|_{2 s}} .
$$

For $0<\rho \in \mathbb{R}$, let $\mathbb{D}_{\rho s}^{d \times r}$ be the set of $\hat{F}$ s, which are described by (8) with $\|\hat{F}\|_{\infty s}<1 / \rho$, and are stochastically stable with $\mathbf{u}_{f}(t) \equiv \mathbf{0}$.

Lemma 4: (See [15]) The system $\hat{F} \in \mathbb{D}_{\rho s}^{d \times r}$ if there exists a $0<\mathbf{P} \in \mathbb{R}^{b \times b}$ such that

$$
\begin{aligned}
{\left[\begin{array}{cc}
\mathbf{P} & \mathbf{0} \\
\mathbf{0} & \frac{1}{\rho^{2}} \mathbf{I}_{r}
\end{array}\right]-} & \sum_{j=1}^{M} q_{j}\left[\begin{array}{cc}
\mathbf{A}_{f}(j) & \mathbf{B}_{f}(j) \\
\mathbf{C}_{f}(j) & \mathbf{D}_{f}(j)
\end{array}\right]^{\mathrm{T}}\left[\begin{array}{cc}
\mathbf{P} & \mathbf{0} \\
\mathbf{0} & \mathbf{I}_{d}
\end{array}\right] \\
& \times\left[\begin{array}{ll}
\mathbf{A}_{f}(j) & \mathbf{B}_{f}(j) \\
\mathbf{C}_{f}(j) & \mathbf{D}_{f}(j)
\end{array}\right]>0
\end{aligned}
$$

Consider a special case of $\hat{F}$ described by

$$
\left\{\begin{aligned}
\mathbf{x}_{f}(t+1)= & \mathbf{A}_{f}\left(\theta_{t}\right) \mathbf{x}_{f}(t)+\mathbf{B}_{f 1}\left(\theta_{t}\right) \mathbf{u}_{f 1}(t) \\
& +\mathbf{B}_{f 2}\left(\theta_{t}\right) \mathbf{u}_{f 2}(t) \\
\mathbf{y}_{f}(t)= & \mathbf{C}_{f}\left(\theta_{t}\right) \mathbf{x}_{f}(t)+\mathbf{D}_{f 1}\left(\theta_{t}\right) \mathbf{u}_{f 1}(t)
\end{aligned}\right.
$$

with $t \in \mathbb{N}$, where $\mathbf{B}_{f 1}, \mathbf{B}_{f 2}$ and $\mathbf{D}_{f 1}$ are mappings from $\mathcal{M}$ to $\mathbb{R}^{b \times r_{1}}, \mathbb{R}^{b \times r_{2}}$ and $\mathbb{R}^{d \times r_{1}}$, respectively. Set

$$
\mathbf{u}_{f 1}(t)=-\mathbf{K}_{f 1}\left(\theta_{t}\right) \mathbf{x}_{f}(t)
$$

where $\mathbf{K}_{f 1}$ is a mapping from $\mathcal{M}$ to $\mathbb{R}^{r_{1} \times b}$. A closed-loop stochastic system $\hat{F}_{c}$ is formed as

$$
\left\{\begin{aligned}
\mathbf{x}_{f}(t+1)= & \left(\mathbf{A}_{f}\left(\theta_{t}\right)-\mathbf{B}_{f 1}\left(\theta_{t}\right) \mathbf{K}_{f 1}\left(\theta_{t}\right)\right) \mathbf{x}_{f}(t) \\
& +\mathbf{B}_{f 2}\left(\theta_{t}\right) \mathbf{u}_{f 2}(t) \\
\mathbf{y}_{f}(t)= & \left(\mathbf{C}_{f}\left(\theta_{t}\right)-\mathbf{D}_{f 1}\left(\theta_{t}\right) \mathbf{K}_{f 1}\left(\theta_{t}\right)\right) \mathbf{x}_{f}(t)
\end{aligned}\right.
$$

with $t \in \mathbb{N}$.

Lemma 5: (See [14]) Suppose that $\hat{F}_{c} \in \mathbb{D}_{\rho s}^{d \times r_{2}}$, $\left(\mathbf{C}_{f}\left(\theta_{t}\right), \mathbf{A}_{f}\left(\theta_{t}\right)\right)$ is stochastically detectable and

$$
\left\{\begin{array}{l}
\mathbf{C}_{f}^{\mathrm{T}}(j) \mathbf{D}_{f 1}(j)=\mathbf{0}, \\
\mathbf{D}_{f 1}^{\mathrm{T}}(j) \mathbf{D}_{f 1}(j)=\mathbf{I},
\end{array} \quad j \in \mathcal{M} .\right.
$$

Then there exist $0 \leq \mathbf{X}_{j} \in \mathbb{R}^{b \times b}$ with $j \in \mathcal{M}$, which satisfy, $\forall j \in \mathcal{M}$,

1) $\mathbf{I}-\frac{1}{\rho^{2}} \mathbf{B}_{f 2}^{\mathrm{T}}(j) \sum_{l=1}^{M} q_{l} \mathbf{X}_{l} \mathbf{B}_{f 2}(j)>0$;

2) $\mathbf{X}_{j}=\mathbf{C}_{f}^{\mathrm{T}}(j) \mathbf{C}_{f}(j)+\mathbf{R}_{f 4}^{\mathrm{T}}(j) \sum_{l=1}^{M} q_{l} \mathbf{X}_{l} \mathbf{R}_{f 4}(j)+$ $\mathbf{R}_{f 2}^{\mathrm{T}}(j) \mathbf{R}_{f 2}(j)-\mathbf{R}_{f 3}^{\mathrm{T}}(j) \mathbf{R}_{f 3}(j)$;

3) $\mathbf{R}_{f 4}\left(\theta_{t}\right)$ is stochastically stable; where 


$$
\begin{aligned}
\mathbf{R}_{f 1}(j)= & \mathbf{I}-\frac{1}{\rho^{2}} \mathbf{B}_{f 2}(j) \mathbf{B}_{f 2}^{\mathrm{T}}(j) \sum_{l=1}^{M} q_{l} \mathbf{X}_{l}, \\
\mathbf{R}_{f 2}(j)= & \left(\mathbf{I}+\mathbf{B}_{f 1}^{\mathrm{T}}(j) \sum_{l=1}^{M} q_{l} \mathbf{X}_{l} \mathbf{R}_{f 1}^{-1}(j) \mathbf{B}_{f 1}(j)\right)^{-1} \\
& \times \mathbf{B}_{f 1}^{\mathrm{T}}(j) \sum_{l=1}^{M} q_{l} \mathbf{X}_{l} \mathbf{R}_{f 1}^{-1}(j) \mathbf{A}_{f}(j), \\
\mathbf{R}_{f 3}(j)= & \left(\mathbf{I}-\frac{1}{\rho^{2}} \mathbf{B}_{f 2}^{\mathrm{T}}(j) \sum_{l=1}^{M} q_{l} \mathbf{X}_{l} \mathbf{B}_{f 2}(j)\right)^{-1} \\
\times & \frac{1}{\rho} \mathbf{B}_{f 2}^{\mathrm{T}}(j) \sum_{l=1}^{M} q_{l} \mathbf{X}_{l}\left(\mathbf{A}_{f}(j)-\mathbf{B}_{f 1}(j) \mathbf{R}_{f 2}(j)\right), \\
\mathbf{R}_{f 4}(j)= & \mathbf{A}_{f}(j)-\mathbf{B}_{f 1}(j) \mathbf{R}_{f 2}(j)+\frac{1}{\rho} \mathbf{B}_{f 2}(j) \mathbf{R}_{f 3}(j) .
\end{aligned}
$$

Finally, consider $\hat{F} \in \mathbb{D}_{\rho s}^{d \times r}$ with $\mathbf{D}_{f}\left(\theta_{t}\right) \equiv \mathbf{0}$, which is obviously equivalent to the system

$$
\left\{\begin{array}{l}
\mathbf{x}_{f}(t+1)=\mathbf{A}_{f}\left(\theta_{t}\right) \mathbf{x}_{f}(t)+\mathbf{B}_{f}\left(\theta_{t}\right) \mathbf{u}_{f}(t), \\
{\left[\begin{array}{c}
\mathbf{y}_{f}(t) \\
0 \mathbf{x}_{f}(t)
\end{array}\right]=\left[\begin{array}{c}
\mathbf{C}_{f}\left(\theta_{t}\right) \\
0 \mathbf{I}_{b}
\end{array}\right] \mathbf{x}_{f}(t),}
\end{array}\right.
$$

Since the above system can be viewed as

$$
\left\{\begin{aligned}
\mathbf{x}_{f}(t+1)= & \mathbf{A}_{f}\left(\theta_{t}\right) \mathbf{x}_{f}(t)+0 \mathbf{I}_{b} \mathbf{w}_{f}(t) \\
& +\mathbf{B}_{f}\left(\theta_{t}\right) \mathbf{u}_{f}(t), \\
{\left[\begin{array}{c}
\mathbf{y}_{f}(t) \\
\mathbf{w}_{f}(t)
\end{array}\right]=} & {\left[\begin{array}{c}
\mathbf{C}_{f}\left(\theta_{t}\right) \\
0 \mathbf{I}_{b}
\end{array}\right] \mathbf{x}_{f}(t) \quad t \in \mathbb{N}, } \\
& +\left[\begin{array}{c}
0 \mathbf{C}_{f}\left(\theta_{t}\right) \\
\mathbf{I}_{b}
\end{array}\right] \mathbf{w}_{f}(t),
\end{aligned}\right.
$$

by connecting $\mathbf{w}_{f}(t)=-\mathbf{K}_{f}\left(\theta_{t}\right) \mathbf{x}_{f}(t)$ with $\mathbf{K}_{f}\left(\theta_{t}\right) \equiv 0 \mathbf{I}_{b}$, it belongs to $\mathbb{D}_{\rho s}^{(d+b) \times r}$. It is easy to check

$$
\begin{aligned}
& {\left[\begin{array}{c}
\mathbf{C}_{f}\left(\theta_{t}\right) \\
0 \mathbf{I}_{b}
\end{array}\right]^{\mathrm{T}}\left[\begin{array}{c}
0 \mathbf{C}_{f}\left(\theta_{t}\right) \\
\mathbf{I}_{b}
\end{array}\right]=\mathbf{0},} \\
& {\left[\begin{array}{c}
0 \mathbf{C}_{f}\left(\theta_{t}\right) \\
\mathbf{I}_{b}
\end{array}\right]^{\mathrm{T}}\left[\begin{array}{c}
0 \mathbf{C}_{f}\left(\theta_{t}\right) \\
\mathbf{I}_{b}
\end{array}\right]=\mathbf{I}_{b} .}
\end{aligned}
$$

Moreover, $\hat{F} \in \mathbb{D}_{\rho s}^{d \times r}$ implies $\mathbf{A}_{f}\left(\theta_{t}\right)$ is stochastically stable and hence $\left(\left[\begin{array}{ll}\mathbf{C}_{f}^{\mathrm{T}}\left(\theta_{t}\right) & 0 \mathbf{I}_{b}\end{array}\right]^{\mathrm{T}}, \mathbf{A}_{f}\left(\theta_{t}\right)\right)$ is stochastically detectable. Thus, applying Lemma 5 to (17) leads to the following corollary.

Corollary 2: Suppose that $\hat{F} \in \mathbb{D}_{\rho s}^{d \times r}$ with $\mathbf{D}_{f}\left(\theta_{t}\right) \equiv \mathbf{0}$. Then there exist $0 \leq \mathbf{X}_{j} \in \mathbb{R}^{b \times b}$ with $j \in \mathcal{M}$, which satisfy, $\forall j \in \mathcal{M}$,

1) $\mathbf{I}-\frac{1}{\rho^{2}} \mathbf{B}_{f}^{\mathrm{T}}(j) \sum_{l=1}^{M} q_{l} \mathbf{X}_{l} \mathbf{B}_{f}(j)>0$;

2) $\mathbf{X}_{j}=\mathbf{C}_{f}^{\mathrm{T}}(j) \mathbf{C}_{f}(j)+\mathbf{S}_{f}^{\mathrm{T}}(j) \sum_{l=1}^{M} q_{l} \mathbf{X}_{l} \mathbf{S}_{f}(j)-$ $\mathbf{R}_{f}^{\mathrm{T}}(j) \mathbf{R}_{f}(j)$

3) $\mathbf{S}_{f}\left(\theta_{t}\right)$ is stochastically stable; where

$$
\begin{aligned}
\mathbf{R}_{f}(j)= & \left(\mathbf{I}-\frac{1}{\rho^{2}} \mathbf{B}_{f}^{\mathrm{T}}(j) \sum_{l=1}^{M} q_{l} \mathbf{X}_{l} \mathbf{B}_{f}(j)\right)^{-1} \\
& \times \frac{1}{\rho} \mathbf{B}_{f}^{\mathrm{T}}(j) \sum_{l=1}^{M} q_{l} \mathbf{X}_{l} \mathbf{A}_{f}(j)
\end{aligned}
$$

$$
\mathbf{S}_{f}(j)=\mathbf{A}_{f}(j)+\frac{1}{\rho} \mathbf{B}_{f}(j) \mathbf{R}_{f}(j) .
$$

\section{PROBLEM Formulation}

The NCS $\hat{P}_{K}$ of Fig. 1 contains a linear time-invariant discrete-time plant $\hat{P}$ and a discrete-time controller $\hat{K}$. The plant $\hat{P}$ consists of a nominal plant model $\hat{P}_{0}$ and an additive perturbation $\hat{\Delta}$, as shown in Fig. 1. $\hat{P}_{0}$ is described by

$$
\left\{\begin{aligned}
\mathbf{x}(t+1) & =\mathbf{A} \mathbf{x}(t)+\mathbf{B u}(t), \\
\mathbf{y}_{0}(t) & =\mathbf{C x}(t)+\mathbf{D} \mathbf{u}(t),
\end{aligned} \quad t \in \mathbb{N}\right.
$$

where $\mathbf{A} \in \mathbb{R}^{n \times n}, \mathbf{B} \in \mathbb{R}^{n \times m}, \mathbf{C} \in \mathbb{R}^{p \times n}$ and $\mathbf{D} \in \mathbb{R}^{p \times m}$ are given matrices. $\hat{\Delta} \in \mathbb{D}_{\gamma}^{p \times m}$ with a given $0<\gamma \in \mathbb{R} . \hat{\Delta}$ and $\hat{P}_{0}$ have the same input $\mathbf{u}(t)$ which is also the input of $\hat{P}$. The output of $\hat{\Delta}$ is added with $\mathbf{y}_{0}(t)$ to form the output of $\hat{P}: \mathbf{y}(t)=\mathbf{y}_{0}(t)+\mathbf{w}(t) . \hat{P}$ and $\hat{K}$ are connected via a shared network through which the sensor transmits data to the controller. The controller is collocated with the actuator. At each instant $t \in \mathbb{N}$, the sensor transmits $\mathbf{y}(t)$ to $\hat{K}$. After the attempt, $\mathbf{y}(t)$ is discarded by the sensor. Each transmission have two alternative outcomes: one is that the transmission succeeds and $\hat{K}$ receives $\mathbf{y}(t)$ at $t$; the other is that the transmission fails due to packet dropout by the network and thus $\hat{K}$ misses $\mathbf{y}(t)$. A successful packet transmission time through the network is assumed to be negligible. A packet arriving late due to network delay has the same effect as packet dropout and is treated as a transmission failure. Those instants at which transmissions succeed are denoted by $t_{k}, k \in \mathbb{N}$, in ascending order, and $t_{0}=0$ is assumed without loss of generality. The time instant $t_{k}$ is referred to as update instant. After $t_{k}, \mathbf{y}\left(t_{k}\right)$ remains to be the newest information for $\hat{K}$ until $t_{k+1}$ when $\mathbf{y}\left(t_{k+1}\right)$ arrives.

It is clear that $\hat{P}_{K}$ is in the mode of open loop when $t \neq t_{k}$ and in the mode of closed loop when $t=t_{k}$. A smart control mechanism, similar to the one in [9], is adopted for $\hat{K}$ as

$$
\left\{\begin{array}{rlrl}
\mathbf{x}_{e}(t+1) & =\mathbf{A} \mathbf{x}_{e}(t)+\mathbf{B u}(t) & \\
+\mathbf{L}\left(\mathbf{C} \mathbf{x}_{e}(t)+\mathbf{D u}(t)-\mathbf{y}(t)\right), & & t=t_{k} \\
\mathbf{x}_{e}(t+1) & =\mathbf{A} \mathbf{x}_{e}(t)+\mathbf{B u}(t), & & t \neq t_{k} \\
\mathbf{u}(t) & =\mathbf{K} \mathbf{x}_{e}(t), & & t \in \mathbb{N}
\end{array}\right.
$$

where the state feedback gain matrix $\mathbf{K} \in \mathbb{R}^{m \times n}$ and the observer gain matrix $\mathbf{L} \in \mathbb{R}^{n \times p}$. When $\mathbf{y}(t)$ is available at $t=t_{k}$, a standard observer law is employed to estimate $\mathbf{x}(t)$

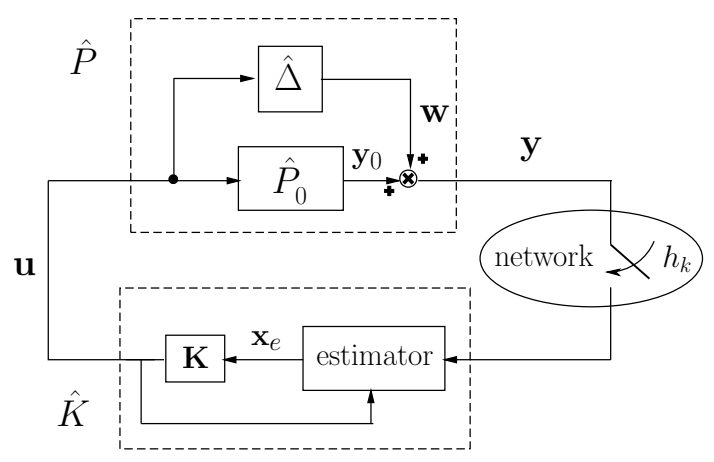

Fig. 1. Networked control system $\hat{P}_{K}$. 
using $\mathbf{x}_{e}(t)$, while when $\mathbf{y}(t)$ is unavailable at $t \neq t_{k}$, an imitation law is employed to estimate $\mathbf{x}(t)$ with $\mathbf{x}_{e}(t)$. Define the update interval

$$
h_{k} \triangleq t_{k+1}-t_{k}, k \in \mathbb{N} .
$$

Let the maximal update interval be $N$, and denote $\mathcal{N}=$ $\{1, \cdots, N\}$. The value of $N$ can be viewed as a network service quality measure. The update intervals $h_{k} \mathrm{~s}$ are assumed to be i.i.d. $\mathcal{N}$-valued random variables. The probability mass function of $h_{k}$ is denoted by $p_{i}=\operatorname{Pr}\left(h_{k}=i\right)$ with $i \in \mathcal{N}$. Let the order of $\hat{\Delta}$ be $q$. Then $\hat{\Delta}$ can be described by

$$
\left\{\begin{aligned}
\mathbf{x}_{\delta}(t+1) & =\mathbf{A}_{\delta} \mathbf{x}_{\delta}(t)+\mathbf{B}_{\delta} \mathbf{u}(t), \\
\mathbf{w}(t) & =\mathbf{C}_{\delta} \mathbf{x}_{\delta}(t)+\mathbf{D}_{\delta} \mathbf{u}(t),
\end{aligned} \quad t \in \mathbb{N}\right.
$$

where the matrices $\mathbf{A}_{\delta} \in \mathbb{R}^{q \times q}, \mathbf{B}_{\delta} \in \mathbb{R}^{q \times m}, \mathbf{C}_{\delta} \in \mathbb{R}^{p \times q}$ and $\mathbf{D}_{\delta} \in \mathbb{R}^{p \times m}$. Define the state of $\hat{P}_{K}$ as

$$
\mathbf{z}(t) \triangleq\left[\mathbf{x}^{\mathrm{T}}(t) \mathbf{x}_{e}^{\mathrm{T}}(t) \mathbf{x}_{\delta}^{\mathrm{T}}(t)\right]^{\mathrm{T}} .
$$

Definition 1: $\hat{P}_{K}$ is mean square stable if $\forall \mathbf{z}(0) \in \mathbb{R}^{2 n+q}$, $\lim _{t \rightarrow \infty} E\left(\mathbf{z}^{\mathrm{T}}(t) \mathbf{z}(t)\right)=0$.

Our NCS design problem can now be stated: given $\mathbf{A}, \mathbf{B}$, $\mathbf{C}, \mathbf{D}, \gamma, N$ and $p_{i} \forall i \in \mathcal{N}$, determine $\mathbf{K}$ and $\mathbf{L}$ such that $\forall \hat{\Delta} \in \mathbb{D}_{\gamma}^{p \times m}, \hat{P}_{K}$ is mean square stable.

\section{Theoretical Analysis and Design Method}

We now study the dynamic response of $\hat{P}_{K}$ by oversampling it at each update instant. Since $h_{k}$ is a $\mathcal{N}$-valued random variable, the dimension of the input (output) of the oversampled system is also random. To tackle this difficulty, we use the auxiliary systems of $\hat{P}_{0}, \hat{\Delta}$ and $\hat{K}$ by augmenting them up to the constant dimension $N m$. The auxiliary system $\hat{P}_{0 s}$ of $\hat{P}_{0}$ is constructed as

$$
\left\{\begin{array}{c}
\overline{\mathbf{x}}(k+1)=\mathbf{A}^{h_{k}} \overline{\mathbf{x}}(k) \\
+\mathcal{W}_{N m}\left(\left[\mathbf{A}^{h_{k}-1} \mathbf{B} \cdots \mathbf{B}\right]\right) \overline{\mathbf{u}}(k), \quad k \in \mathbb{N} . \\
\overline{\mathbf{y}}_{0}(k)=\mathbf{C} \overline{\mathbf{x}}(k)+\mathcal{W}_{N m}(\mathbf{D}) \overline{\mathbf{u}}(k),
\end{array}\right.
$$

The auxiliary system $\hat{\Delta}_{s}$ of $\hat{\Delta}$ is constructed as

$$
\left\{\begin{array}{l}
\overline{\mathbf{x}}_{\delta}(k+1)=\mathbf{A}_{\delta}^{h_{k}} \overline{\mathbf{x}}_{\delta}(k) \\
\quad+\mathcal{W}_{N m}\left(\left[\mathbf{A}_{\delta}^{h_{k}-1} \mathbf{B}_{\delta} \cdots \mathbf{B}_{\delta}\right]\right) \overline{\mathbf{u}}(k), \quad k \in \mathbb{N} . \\
\overline{\mathbf{w}}(k)=\mathbf{C}_{\delta} \overline{\mathbf{x}}_{\delta}(k)+\mathcal{W}_{N m}\left(\mathbf{D}_{\delta}\right) \overline{\mathbf{u}}(k),
\end{array}\right.
$$

The auxiliary system $\hat{K}_{s}$ of $\hat{K}$ is constructed as

$$
\left\{\begin{array}{c}
\overline{\mathbf{x}}_{e}(k+1)=\boldsymbol{\Lambda}_{0}^{h_{k}-1} \boldsymbol{\Lambda}_{1} \overline{\mathbf{x}}_{e}(k) \\
-\boldsymbol{\Lambda}_{0}^{h_{k}-1} \mathbf{L} \overline{\mathbf{y}}_{0}(k)-\boldsymbol{\Lambda}_{0}^{h_{k}-1} \mathbf{L} \overline{\mathbf{w}}(k), \quad k \in \mathbb{N} \\
\overline{\mathbf{u}}(k)=\boldsymbol{\Upsilon}_{1}\left(h_{k}\right) \overline{\mathbf{x}}_{e}(k) \\
-\mathbf{\Upsilon}_{2}\left(h_{k}\right) \overline{\mathbf{y}}_{0}(k)-\boldsymbol{\Upsilon}_{2}\left(h_{k}\right) \overline{\mathbf{w}}(k),
\end{array}\right.
$$

where $\boldsymbol{\Lambda}_{0}=\mathbf{A}+\mathbf{B K}, \boldsymbol{\Lambda}_{1}=\mathbf{A}+\mathbf{B K}+\mathbf{L C}+\mathbf{L D K}$,

$$
\begin{gathered}
\boldsymbol{\Upsilon}_{1}\left(h_{k}\right)=\left\{\begin{array}{cc}
\mathcal{H}_{N m}(\mathbf{K}), & h_{k}=1, \\
\mathcal{H}_{N m}\left(\left[\mathbf{K}^{\mathrm{T}}\left(\mathbf{K} \mathbf{\Lambda}_{1}\right)^{\mathrm{T}} \ldots\right.\right. & \\
\left.\left.\left(\mathbf{K} \boldsymbol{\Lambda}_{0}^{h_{k}-2} \boldsymbol{\Lambda}_{1}\right)^{\mathrm{T}}\right]^{\mathrm{T}}\right), & h_{k}>1,
\end{array}\right. \\
\boldsymbol{\Upsilon}_{2}\left(h_{k}\right)=\left\{\begin{array}{cc}
\mathcal{H}_{N m}(\mathbf{O K L}), & h_{k}=1, \\
\mathcal{H}_{N m}\left(\left[(\mathbf{O K L})^{\mathrm{T}}(\mathbf{K L})^{\mathrm{T}} \ldots\right.\right. & \\
\left.\left.\left(\mathbf{K} \boldsymbol{\Lambda}_{0}^{h_{k}-2} \mathbf{L}\right)^{\mathrm{T}}\right]^{\mathrm{T}}\right), & h_{k}>1 .
\end{array}\right.
\end{gathered}
$$

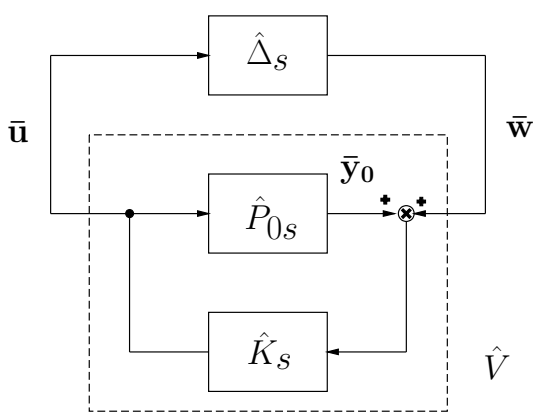

Fig. 2. Auxiliary system $\hat{P}_{K s}$ for $\hat{P}_{K}$.

Combining $\hat{P}_{0 s}, \hat{\Delta}_{s}$ and $\hat{K}_{s}$ forms the auxiliary stochastic system $\hat{P}_{K s}$, depicted in Fig. 2, of $\hat{P}_{K}$. Define

$$
\overline{\mathbf{z}}(k)=\left[\overline{\mathbf{x}}^{\mathrm{T}}(k) \overline{\mathbf{x}}_{e}^{\mathrm{T}}(k) \overline{\mathbf{x}}_{\delta}^{\mathrm{T}}(k)\right]^{\mathrm{T}} .
$$

From (23) to (33), we have the following relationships between $\hat{P}_{K s}$ and $\hat{P}_{K}$. Given $t_{0}=0$ and $\overline{\mathbf{z}}(0)=\mathbf{z}\left(t_{0}\right)$, $\forall k \in \mathbb{N}$,

$$
\begin{aligned}
\overline{\mathbf{z}}(k) & =\mathbf{z}\left(t_{k}\right), \\
\overline{\mathbf{y}}_{0}(k) & =\mathbf{y}_{0}\left(t_{k}\right), \\
\overline{\mathbf{w}}(k) & =\mathbf{w}\left(t_{k}\right), \\
\overline{\mathbf{u}}(k) & =\mathcal{H}_{N m}\left(\left[\mathbf{u}^{\mathrm{T}}\left(t_{k}\right) \cdots \mathbf{u}^{\mathrm{T}}\left(t_{k}+h_{k}-1\right)\right]^{\mathrm{T}}\right) .
\end{aligned}
$$

These results imply that $\lim _{k \rightarrow \infty} E\left(\overline{\mathbf{z}}^{\mathrm{T}}(k) \overline{\mathbf{z}}(k)\right)=0$ if $\lim _{t \rightarrow \infty} E\left(\mathbf{z}^{\mathrm{T}}(t) \mathbf{z}(t)\right)=0$. On the other hand, as $h_{k}$ is bounded by $N$, there exists a constant real scalar $\eta$ independent of $k$ and $\tau$ such that $\forall k \in \mathbb{N}, \mathbf{z}^{\mathrm{T}}\left(t_{k}+\tau\right) \mathbf{z}\left(t_{k}+\tau\right)<$ $\eta \mathbf{z}^{\mathrm{T}}\left(t_{k}\right) \mathbf{z}\left(t_{k}\right)$ for any $\tau \in\left\{1, \cdots, h_{k}\right\}$. Then (34) implies that $\lim _{t \rightarrow \infty} E\left(\mathbf{z}^{\mathrm{T}}(t) \mathbf{z}(t)\right)=0$ if $\lim _{k \rightarrow \infty} E\left(\overline{\mathbf{z}}^{\mathrm{T}}(k) \overline{\mathbf{z}}(k)\right)=0$. Thus, we have the following proposition.

Proposition 1: $\hat{P}_{K}$ is mean square stable if and only if $\hat{P}_{K s}$ is stochastically stable.

Next, we discuss the relationship between $\hat{\Delta}$ and $\hat{\Delta}_{s}$.

Proposition 2: For any $\hat{\Delta} \in \mathbb{D}_{\gamma}^{p \times m}$, its auxiliary system $\hat{\Delta}_{s} \in \mathbb{D}_{\gamma s}^{p \times(N m)}$.

Proof: From $\hat{\Delta} \in \mathbb{D}_{\gamma}^{p \times m}$ and Lemma 1 , there exist $0<$ $\mathbf{P}_{\delta} \in \mathbb{R}^{q \times q}$ such that

$$
\left[\begin{array}{cc}
\mathbf{P}_{\delta} & \mathbf{0} \\
\mathbf{0} & \frac{1}{\gamma^{2}} \mathbf{I}_{m}
\end{array}\right]-\left[\begin{array}{cc}
\mathbf{A}_{\delta} & \mathbf{B}_{\delta} \\
\mathbf{C}_{\delta} & \mathbf{D}_{\delta}
\end{array}\right]^{\mathrm{T}}\left[\begin{array}{cc}
\mathbf{P}_{\delta} & \mathbf{0} \\
\mathbf{0} & \mathbf{I}_{p}
\end{array}\right]\left[\begin{array}{cc}
\mathbf{A}_{\delta} & \mathbf{B}_{\delta} \\
\mathbf{C}_{\delta} & \mathbf{D}_{\delta}
\end{array}\right]
$$$$
>0 \text {. }
$$

Noticing (26), inequality (38) means that $\forall t \in \mathbb{N}$ and $\forall\left[\begin{array}{ll}\mathbf{x}_{\delta}^{\mathrm{T}}(t) & \mathbf{u}^{\mathrm{T}}(t)\end{array}\right]^{\mathrm{T}} \in \mathbb{R}^{q+m}$,

$$
\begin{aligned}
\mathbf{x}_{\delta}^{\mathrm{T}}(t) & \mathbf{P}_{\delta} \mathbf{x}_{\delta}(t)+\frac{1}{\gamma^{2}} \mathbf{u}^{\mathrm{T}}(t) \mathbf{u}(t) \geq \\
& \mathbf{w}{ }^{\mathrm{T}}(t) \mathbf{w}(t)+\mathbf{x}_{\delta}^{\mathrm{T}}(t+1) \mathbf{P}_{\delta} \mathbf{x}_{\delta}(t+1) .
\end{aligned}
$$

Equality holds in (39) if and only if $\left[\mathbf{x}_{\delta}^{\mathrm{T}}(t) \mathbf{u}^{\mathrm{T}}(t)\right]^{\mathrm{T}}=\mathbf{0}$. Now $\forall t \in \mathbb{N}, \forall i \in \mathcal{N}$ and $\forall\left[\mathbf{x}_{\delta}^{\mathrm{T}}(t) \mathbf{u}^{\mathrm{T}}(t) \cdots \mathbf{u}^{\mathrm{T}}(t+i-1)\right]^{\mathrm{T}} \in$ $\mathbb{R}^{q+i m}$,

$$
\mathbf{x}_{\delta}^{\mathrm{T}}(t) \mathbf{P}_{\delta} \mathbf{x}_{\delta}(t)+\frac{1}{\gamma^{2}} \sum_{l=0}^{i-1} \mathbf{u}^{\mathrm{T}}(t+l) \mathbf{u}(t+l) \geq \mathbf{w}^{\mathrm{T}}(t) \mathbf{w}(t)
$$




$$
\begin{gathered}
+\mathbf{x}_{\delta}^{\mathrm{T}}(t+1) \mathbf{P}_{\delta} \mathbf{x}_{\delta}(t+1)+\frac{1}{\gamma^{2}} \sum_{l=1}^{i-1} \mathbf{u}^{\mathrm{T}}(t+l) \mathbf{u}(t+l) \geq \\
\sum_{l=0}^{i-1} \mathbf{w}^{\mathrm{T}}(t+l) \mathbf{w}(t+l)+\mathbf{x}_{\delta}^{\mathrm{T}}(t+i) \mathbf{P}_{\delta} \mathbf{x}_{\delta}(t+i) \geq \\
\mathbf{w}^{\mathrm{T}}(t) \mathbf{w}(t)+\mathbf{x}_{\delta}^{\mathrm{T}}(t+i) \mathbf{P}_{\delta} \mathbf{x}_{\delta}(t+i) .
\end{gathered}
$$

Equality holds in (40) if and only if $\left[\mathbf{x}_{\delta}^{\mathrm{T}}(t) \mathbf{u}^{\mathrm{T}}(t) \cdots \mathbf{u}^{\mathrm{T}}(t+\right.$ $i-1)]^{\mathrm{T}}=\mathbf{0}$. Since

$$
\begin{aligned}
& {\left[\begin{array}{c}
\mathbf{x}_{\delta}(t+i) \\
\mathbf{w}(t)
\end{array}\right]=} {\left[\begin{array}{cccc}
\mathbf{A}_{\delta}^{i} & \mathbf{A}_{\delta}^{i-1} \mathbf{B}_{\delta} & \cdots & \mathbf{B}_{\delta} \\
\mathbf{C}_{\delta} & \mathbf{D}_{\delta} & \mathbf{0}
\end{array}\right] } \\
& \times\left[\begin{array}{c}
\mathbf{x}_{\delta}(t) \\
\mathbf{u}(t) \\
\vdots \\
\mathbf{u}(t+i-1)
\end{array}\right]
\end{aligned}
$$

Inequality (40) means that $\forall i \in \mathcal{N}$,

$$
\begin{aligned}
& {\left[\begin{array}{cc}
\mathbf{P}_{\delta} & \mathbf{0} \\
\mathbf{0} & \frac{1}{\gamma^{2}} \mathbf{I}_{i m}
\end{array}\right]-\left[\begin{array}{cc}
\left(\mathbf{A}_{\delta}^{\mathrm{T}}\right)^{i} & \mathbf{C}_{\delta}^{\mathrm{T}} \\
\mathbf{B}_{\delta}^{\mathrm{T}}\left(\mathbf{A}_{\delta}^{\mathrm{T}}\right)^{i-1} & \mathbf{D}_{\delta}^{\mathrm{T}} \\
\vdots & \mathbf{0}
\end{array}\right]\left[\begin{array}{cc}
\mathbf{P}_{\delta} & \mathbf{0} \\
\mathbf{0} & \mathbf{I}_{p}
\end{array}\right]} \\
& \times\left[\begin{array}{cccc}
\mathbf{A}_{\delta}^{i} & \mathbf{A}_{\delta}^{i-1} \mathbf{B}_{\delta} & \cdots & \mathbf{B}_{\delta} \\
\mathbf{C}_{\delta} & \mathbf{D}_{\delta} & \multicolumn{0}{c}{}
\end{array}\right]>0 .
\end{aligned}
$$

Hence

$$
\left[\begin{array}{cc}
\mathbf{P}_{\delta} & \mathbf{0} \\
\mathbf{0} & \frac{1}{\gamma^{2}} \mathbf{I}_{N m}
\end{array}\right]-\sum_{i=1}^{N} p_{i} \boldsymbol{\Phi}_{i}^{\mathrm{T}}\left[\begin{array}{cc}
\mathbf{P}_{\delta} & \mathbf{0} \\
\mathbf{0} & \mathbf{I}_{p}
\end{array}\right] \boldsymbol{\Phi}_{i}>0
$$

with

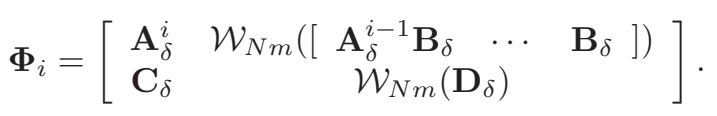

Applying Lemma 4 to (43) completes the proof.

From Propositions 1 and 2, we have the following result.

Proposition 3: Suppose that $\forall \hat{\Delta}_{s} \in \mathbb{D}_{\gamma s}^{p \times(N m)}, \hat{P}_{K s}$ is stochastically stable. Then $\forall \hat{\Delta} \in \mathbb{D}_{\gamma}^{p \times m}, \hat{P}_{K}$ is mean square stable.

Let $\hat{P}_{K s}$ be divided into an unknown part $\hat{\Delta}_{s}$ and a known part $\hat{V}$, as shown in Fig. 2. $\hat{V}$ is the closed-loop system formed by $\hat{P}_{0 s}$ and $\hat{K}_{s}$, and is described as

$$
\left\{\begin{array}{c}
{\left[\begin{array}{c}
\overline{\mathbf{x}}(k+1) \\
\overline{\mathbf{x}}_{e}(k+1)
\end{array}\right]=\mathbf{A}_{v}\left(h_{k}\right)\left[\begin{array}{c}
\overline{\mathbf{x}}(k) \\
\overline{\mathbf{x}}_{e}(k)
\end{array}\right]} \\
+\mathbf{B}_{v}\left(h_{k}\right) \overline{\mathbf{w}}(k), \quad k \in \mathbb{N} \\
\overline{\mathbf{u}}(k)=\mathbf{C}_{v}\left(h_{k}\right)\left[\begin{array}{c}
\overline{\mathbf{x}}(k) \\
\overline{\mathbf{x}}_{e}(k)
\end{array}\right]+\mathbf{D}_{v}\left(h_{k}\right) \overline{\mathbf{w}}(k),
\end{array}\right.
$$

where

$$
\begin{aligned}
& \mathbf{A}_{v}\left(h_{k}\right)=\left[\begin{array}{cc}
\mathbf{A} & \mathbf{B K} \\
\mathbf{0} & \boldsymbol{\Lambda}_{0}
\end{array}\right]^{h_{k}-1}\left[\begin{array}{cc}
\mathbf{A} & \mathbf{B K} \\
-\mathbf{L} \mathbf{C} & \boldsymbol{\Lambda}_{0}+\mathbf{L} \mathbf{C}
\end{array}\right], \\
& \mathbf{B}_{v}\left(h_{k}\right)=\left[\begin{array}{cc}
\mathbf{A} & \mathbf{B K} \\
\mathbf{0} & \boldsymbol{\Lambda}_{0}
\end{array}\right]^{h_{k}-1}\left[\begin{array}{c}
\mathbf{0} \\
-\mathbf{L}
\end{array}\right] \\
& \mathbf{C}_{v}\left(h_{k}\right)=\left[\begin{array}{ll}
-\mathbf{\Upsilon}_{2}\left(h_{k}\right) \mathbf{C} & \mathbf{\Upsilon}_{3}\left(h_{k}\right)
\end{array}\right] \\
& \mathbf{D}_{v}\left(h_{k}\right)=-\mathbf{\Upsilon}_{2}\left(h_{k}\right)
\end{aligned}
$$

with

$$
\boldsymbol{\Upsilon}_{3}\left(h_{k}\right)= \begin{cases}\mathcal{H}_{N m}(\mathbf{K}), & h_{k}=1, \\ \mathcal{H}_{N m}\left(\left[\mathbf{K}^{\mathrm{T}}\left(\mathbf{K}\left(\boldsymbol{\Lambda}_{0}+\mathbf{L} \mathbf{C}\right)\right)^{\mathrm{T}}\right.\right. & \\ \left.\left.\cdots\left(\mathbf{K} \boldsymbol{\Lambda}_{0}^{h_{k}-2}\left(\boldsymbol{\Lambda}_{0}+\mathbf{L} \mathbf{C}\right)\right)^{\mathrm{T}}\right]^{\mathrm{T}}\right), h_{k}>1 .\end{cases}
$$

Let $\xi=1 / \gamma$. Then our main result can be presented.

Theorem 1: Suppose that $\hat{V} \in \mathbb{D}_{\xi s}^{(N m) \times p}$. Then $\forall \hat{\Delta}_{s} \in$ $\mathbb{D}_{\gamma s}^{p \times(N m)}, \hat{P}_{K s}$ is stochastically stable.

Proof: The proof is cut off due to space limitation. Contact the authors for the detailed proof.

We refer to Theorem 1 as the small gain theorem of discretetime stochastic systems. Note that the small gain theorem of [19] is valid for deterministic systems while the small gain theorem of [21] is derived for continuous-time stochastic systems.

According to Proposition 3 and Theorem 1, any pair of $\mathbf{K}$ and $\mathbf{L}$ ensuring $\hat{V} \in \mathbb{D}_{\xi s}^{(N m) \times p}$ is a solution to our NCS design problem. Since $\mathbf{A}, \mathbf{B}, \mathbf{C}, \mathbf{D}, \gamma, N$ and $p_{i}, \forall i \in \mathcal{N}$, are known, $\mathbf{A}_{v}(i), \mathbf{B}_{v}(i), \mathbf{C}_{v}(i)$ and $\mathbf{D}_{v}(i)$ with $i \in \mathcal{N}$ in (46) to (49) are functions of $\mathbf{K}$ and $\mathbf{L}$. Therefore, we can denote

$$
\mathbf{F}_{i}(\mathbf{K}, \mathbf{L})=\left[\begin{array}{ll}
\mathbf{A}_{v}(i) & \mathbf{B}_{v}(i) \\
\mathbf{C}_{v}(i) & \mathbf{D}_{v}(i)
\end{array}\right], i \in \mathcal{N}
$$

Further define

$$
\begin{aligned}
\underline{\alpha}(\mathbf{K}, \mathbf{L})= & \inf _{\substack{0<\mathbf{Q} \in \mathbb{R}^{(2 n) \times(2 n)} \\
\alpha \in \mathbb{R}}}\{\alpha \mid \alpha \mathbf{U}(\mathbf{Q}, \gamma)> \\
& \left.\sum_{i=1}^{N} p_{i} \mathbf{F}_{i}^{\mathrm{T}}(\mathbf{K}, \mathbf{L}) \mathbf{S}(\mathbf{Q}) \mathbf{F}_{i}(\mathbf{K}, \mathbf{L})\right\}
\end{aligned}
$$

with

$$
\mathbf{U}(\mathbf{Q}, \gamma)=\left[\begin{array}{cc}
\mathbf{Q} & \mathbf{0} \\
\mathbf{0} & \gamma \mathbf{I}_{p}
\end{array}\right] \text { and } \mathbf{S}(\mathbf{Q})=\left[\begin{array}{cc}
\mathbf{Q} & \mathbf{0} \\
\mathbf{0} & \mathbf{I}_{N m}
\end{array}\right] \text {. }
$$

For given $\mathbf{K} \in \mathbb{R}^{m \times n}$ and $\mathbf{L} \in \mathbb{R}^{n \times p}, \underline{\alpha}(\mathbf{K}, \mathbf{L})$ can be computed conveniently by a combination of linear matrix inequality (LMI) technique [22] and bisection search [23]. From Proposition 3 and Theorem 1 as well as Lemma 4, the following result is plain.

Corollary 3: A pair of $\mathbf{K} \in \mathbb{R}^{m \times n}$ and $\mathbf{L} \in \mathbb{R}^{n \times p}$ guarantee that $\hat{P}_{K}$ is mean square stable for any $\hat{\Delta} \in \mathbb{D}_{\gamma}^{p \times m}$, if $\underline{\alpha}(\mathbf{K}, \mathbf{L})<1$.

According to Corollary 3, we can design $\mathbf{K}$ and $\mathbf{L}$ by solving the nonlinear optimisation problem

$$
\mu=\inf _{\substack{\mathbf{K} \in \mathbb{R}^{m \times n} \\ \mathbf{L} \in \mathbb{R}^{n \times p}}} \underline{\alpha}(\mathbf{K}, \mathbf{L}) .
$$

We solve this optimisation problem using the genetic algorithm (GA) [24] to obtain a pair of $\mathbf{K}^{*}$ and $\mathbf{L}^{*}$ such that $\underline{\alpha}\left(\mathbf{K}^{*}, \mathbf{L}^{*}\right)<1$. Note that in some cases we may be unable to achieve $\underline{\alpha}(\mathbf{K}, \mathbf{L})<1$, even though the NCS design problem does have solutions. This is because Corollary 3 only provides a sufficient condition. If $\mathbf{K}^{*}$ and $\mathbf{L}^{*}$ are not found by the GA to meet $\underline{\alpha}\left(\mathbf{K}^{*}, \mathbf{L}^{*}\right)<1$, we can rearrange some conditions of the NCS design problem, for example by increasing the value of $\gamma$, to ease the design problem. 
(a)

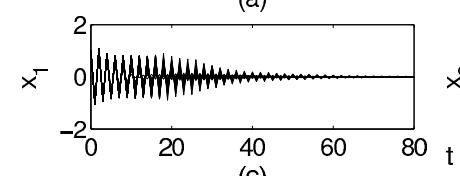

(c)

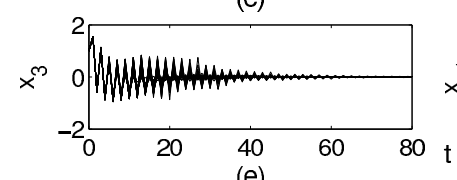

(e)
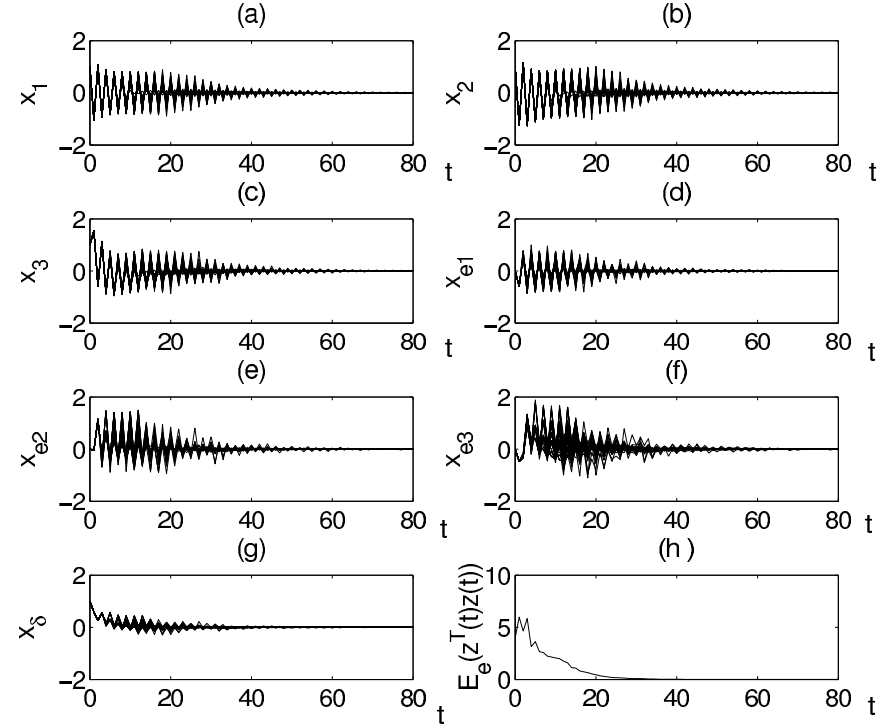

Fig. 3. (a) to (g): state trajectories of the plant $\hat{P}_{K}$ for 50 simulations, and (h): $E_{\mathrm{e}}\left(\mathbf{z}^{\mathrm{T}}(t) \mathbf{z}(t)\right)$ calculated by averaging $\mathbf{z}^{\mathrm{T}}(t) \mathbf{z}(t)$ over these 50 simulations.

\section{An NumericAl EXAMPle}

Based on the method presented in the previous section, a MATLAB program for NCS design was developed where the feasp and ga functions of MATLAB were used to solve LMI and to implement GA, respectively. We considered an unstable third-order $\hat{P}_{0}$ of (23) with the parameters

$$
\begin{gathered}
\mathbf{A}=\left[\begin{array}{ccc}
-1.05 & 0 & 0 \\
-2 & 0.75 & 0 \\
0 & 1.05 & 0.5
\end{array}\right], \mathbf{B}=\left[\begin{array}{c}
0.5 \\
0 \\
0.5
\end{array}\right] \\
\mathbf{C}=\left[\begin{array}{lll}
1 & 1 & 0
\end{array}\right], \mathbf{D}=0.2
\end{gathered}
$$

Given $N=10, p_{i}=0.1$ for $i \in \mathcal{N} \triangleq\{1, \ldots, 10\}$ and $\gamma=0.4$, we solved this NCS design problem to yield $\mathbf{K}^{*}=\left[\begin{array}{lll}-0.2741 & 0.5791 & 0.0260\end{array}\right]$ and $\mathbf{L}^{*}=$ $\left[\begin{array}{lll}0.2657 & 0.1480 & -0.1718\end{array}\right]^{\mathrm{T}}$ with $\underline{\alpha}\left(\mathbf{K}^{*}, \mathbf{L}^{*}\right)=0.9872<1$. The NCS with the designed $\mathbf{K}^{*}$ and $\mathbf{L}^{*}$ was then simulated in the MATLAB platform for 50 times. In the simulation, a stable first-order $\hat{\Delta}$ of (26) was specified by $\mathbf{A}_{\delta}=0.5833, \mathbf{B}_{\delta}=1$, $\mathbf{C}_{\delta}=1$ and $\mathbf{D}_{\delta}=0.1$ with $\|\hat{\Delta}\|_{\infty}=2.4998<1 / \gamma$. The initial state was chosen to be $\mathbf{x}(0)=\left[x_{1}(0) x_{2}(0) x_{3}(0)\right]^{\mathrm{T}}=$ $\left[\begin{array}{lll}1 & 1 & 1\end{array}\right]^{\mathrm{T}}, \mathbf{x}_{e}(0)=\left[\begin{array}{lll}x_{e 1}(0) & x_{e 2}(0) & x_{e 3}(0)\end{array}\right]^{\mathrm{T}}=\left[\begin{array}{lll}0 & 0 & 0\end{array}\right]^{\mathrm{T}}$ and $\mathbf{x}_{\delta}(0)=1$. Fig. 3 (a) to (g) depict the 50 trajectories of each state element, respectively. These trajectories display our NCS behaviour under the 50 different realisations of $\left\{h_{k}\right\}$. For any $t \in \mathbb{N}$, we obtained 50 observations of the random variable $\mathbf{z}^{\mathrm{T}}(t) \mathbf{z}(t)$. The first sample moment of these 50 observations, denoted by $E_{\mathrm{e}}\left(\mathbf{z}^{\mathrm{T}}(t) \mathbf{z}(t)\right)$, was shown in Fig. $3(\mathrm{~h})$.

\section{CONCLUSIONS}

Discrete-time NCSs have been studied where the plant has additive uncertainty and a smart controller is updated with the sensor information at stochastic time intervals. It has been shown that the issue is linked to robust control of linear discrete-time stochastic systems, and a new small gain theorem has been derived under the condition that update intervals are i.i.d. $\mathcal{N}$-valued random variables. Based on this result, sufficient conditions have been established for guaranteering the mean square stability of NCSs and a design method for smart controller has been provided.

\section{REFERENCES}

[1] Y. Tipsuwan and M.Y. Chow, "Control methodologies in networked control systems," Control Eng. Practice, vol.11, no.10, pp.1099-1111, 2003.

[2] J.R. Moyne and D.M. Tilbury, "The emergence of industrial control networks for manufacturing control, diagnostics, and safety data," Proc. IEEE, vol.95, no.1, pp.29-47, 2007.

[3] L. Schenato, B. Sinopoli, M. Franceschetti, K. Poolla and S.S. Sastry, "Foundations of control and estimation over lossy networks," Proc. IEEE, vol.95, no.1, pp.163-187, 2007.

[4] W.-A. Zhang and L. Yu, "Output feedback stabilization of networked control systems with packet dropouts," IEEE Trans. Automatic Control, vol.52, no.9, pp.1705-1710, 2007.

[5] H. Ishii, " $H^{\infty}$ control with limited communication and message losses," Systems and Control Letters, vol.57, no.4, pp.322-331, 2008.

[6] W. Zhang, M.S. Branicky and S.M. Phillips, "Stability of networked control systems," IEEE Control System Magazine, vol.21, no.1, pp.8499, 2001.

[7] L.A. Montestruque and P.J. Antsaklis, "On the model-based control of networked systems," Automatica, vol.39, no.10, pp.1837-1843, 2003.

[8] L.A. Montestruque and P.J. Antsaklis, "Stability of model-based networked control systems with time-varying transmission times," IEEE Trans. Automatic Control, vol.49, no.9, pp.1562-1572, 2004.

[9] P.V. Zhivoglyadov and R.H. Middleton, "Networked control design for linear systems," Automatica, vol.39, no.4, pp.743-750, 2003.

[10] J. Nilsson, B. Bernhardsson and B. Wittenmark, "Stochastic analysis and control of real-time systems with random time delays," Automatica, vol.34, no.1, pp.57-64, 1998.

[11] L. Zhang, Y. Shi, T. Chen and B. Huang, "A new method for stabilization of networked control systems with random delays," IEEE Trans. Automatic Control, vol.50, no.8, pp.1177-1181, 2005.

[12] Y. Ji, H.J. Chizeck, X. Feng and K.A. Loparo, "Stability and control of discrete-time jump linear systems," Control Theory Adv. Technol., vol.7, no.2, pp.247-270, 1991.

[13] O.L.V. Costa and M.D. Fragoso, "Stability results for discrete-time linear systems with Markovian jumping parameters," J. Math. Analysis and Appl., vol.179, no.1, pp.154-178, 1993.

[14] O.L.V. Costa and J.B.R. do Val, "Full information $H_{\infty}$-Control for discrete-time infinite Markov jump parameter systems," J. Math. Analysis and Appl., vol.202, no.2, pp.578-603, 1996.

[15] P. Seiler and R. Sengupta, "An $H_{\infty}$ approach to networked control," IEEE Trans. Automatic Control, vol.50, no.3, pp.356-364, 2005.

[16] N. Elia, "Remote stabilization over fading channels," Systems and Control Letters, vol.54, no.3, pp.237-249, 2005.

[17] S. Hu and W.-Y. Yan, "Stability robustness of networked control systems with respect to packet loss," Automatica, vol.43, no.7, pp.1243-1248, 2007.

[18] D. Yue, Q.-L. Han and J. Lam, "Network-based robust $H_{\infty}$ control of systems with uncertainty," Automatica, vol.41, no.6, pp.999-1007, 2005.

[19] K. Zhou, J.C. Doyle and K. Glover, Robust and Optimal Control. Englewood Cliffs, NJ: Prentice-Hall, 1995.

[20] V. Dragan and T. Morozan, "Exponential stability for discrete time linear equations defined by positive operators," Integral Equ. Oper. Theory, vol.54, pp.465-493, 2006.

[21] V. Dragan, A. Halanay and A. Stoica, "A small gain theorem for linear stochastic systems," Systems and Control Letters, vol.30, pp.243-251, 1997.

[22] S. Boyd, L. El Ghaoui, E. Feron and V. Balakrishan, Linear Matrix Inequalities in Systems and Control Theory. Philadelphia, PA: SIAM, 1994.

[23] A. Quarteroni, R. Sacco and F. Saleri, Numerical Mathematics. New York: Springer-Verlag, 2000.

[24] D.E. Goldberg, Genetic Algorithms in Search, Optimization and Machine Learning. Reading, MA: Addison Wesley, 1989. 